\section{Obstetric difficulties due to Graves' disease}

Q1: Would more aggressive monitoring of the maternal thyroid status and fetal condition in the unsuccessful pregnancies have led to a better outcome?

A more aggressive management of maternal thyroid status is definitely expected to improve the fetal outcome. A higher incidence of congenital malformation, neonatal mortality, and low birthweight infants is seen in untreated Graves' disease. ${ }^{1}$ Hyperthyroid untreated mothers had the highest incidence of fetal anomalies $(6 \%)$ making it clear that treatment and monitoring is of paramount importance. ${ }^{1}$ An equal and low rate $(1 \%)$ of fetal anomalies had been reported to mothers rendered euthyroid by antithyroid drugs and monitored throughout the pregnancy in comparison to euthyroid untreated mothers with Graves' disease. $^{12}$

\section{Q2: Is it likely that the difference in the outcome of those unsuccessful pregnancies was related to the maternal uncontrolled thyroid state or to the circulating antibody levels?}

In one word it is difficult to tell which of the two, untreated thyroid status or high circulating level, is more important. To the authors it looks that both are equally important. Thyroid stimulating immunoglobulins can cross the placenta freely and can affect the developing fetus adversely. Also there is a possibility that after the definitive treatment of the hyperthyroidism with radioactive iodine the antibody titre had dropped, and this had favoured the baby.

Q3: What are the three crucial factors for the successful outcome in this case?

The three crucial factors in the above case are careful planning before conception, close supervision, and joint management.

\section{Discussion}

Graves' disease diagnosed during pregnancy needs careful monitoring, maintaining an euthyroid state with TSH in the normal range. A block and replacement regimen of antithyroid drugs and thyroxine is not an option as thyroxine does not cross the placenta but antithyroid drugs do freely. For obvious reasons radioactive iodine is contraindicated in pregnancy. Therefore antithyroid drugs are the main option. The dose of antithyroid drugs should be as low as possible to keep the free thyroxine in the upper level and serum TSH in the lower level of the normal range. ${ }^{2}$ Most studies show both propylthiouracil and carbimazole can be used in pregnancy. ${ }^{34}$ Although propylthiouracil is preferred, especially in the United States, an advantage has not been proved. In a recently conducted study the incidence of major congenital malformations in

\section{Learning points}

- Thyroid disease in pregnancy is not uncommon.

- Untreated Graves' disease has unfavourable outcome both to mother and the baby.

- Maternal free thyroxine should be kept in the upper normal range.

- Measuring TSHRAb is important to predict neonatal hyperthyroidism.

- Careful planning before conception, close supervision, and joint management is important for a successful outcome.

mothers treated with propylthiouracil and carbimazole was reported to be $3 \%$ and $2.7 \%$ respectively. ${ }^{4}$ As propylthiouracil is more protein bound, it is secreted less in milk and is usually preferred during breast feeding.

The situation is different when Graves' disease is diagnosed before conception. Every effort should be made to make the mother euthyroid before conception. This will obviously reduce the effect of maternal hyperthyroidism on the developing fetus. Even when the mother is clinically and biochemically euthyroid, she may have plenty of TSHRAb that can cross the placenta and affect the baby. In our patient, there was no evidence of maternal hyperthyroidism in the previous pregnancies resulting into stillbirths. It is clear from the postmortem results that stillbirths were due to intrauterine Graves' disease. TSHRAb was not monitored in the previous pregnancies.

Due consideration should be given the fetal thyroid state. The problems encountered are growth retardation, stillbirth, and fetal Graves' disease. All the four risk factors found to be associated with poor maternal-fetal outcome (maternal hyperthyroidism, a history of Graves' disease for more than 10 years, the onset of Graves' disease before the age of 20 years, and positive TSHRAb during pregnancy) were present in our patient. ${ }^{5}$ Fetal Graves' disease is suspected by growth retardation, goitre on ultrasound scan, and fetal tachycardia. It is treated by giving antithyroid drugs to the mother. In cases where the mother is euthyroid, she should be given thyroxine to prevent the development of hypothyroidism from antithyroid medication given for the fetus. The best time to perform subtotal thyroidectomy, if required, is the second trimester. In cases where radioactive iodine is given, the patient should not conceive for at least four months after the radioactive iodine.

In conclusion, untreated/unsupervised Graves' disease in pregnancy can have adverse effects on mother and baby. Our patient, who presented with juvenile hyperthyroidism initially, illustrates the importance of careful supervision of thyroid status during pregnancy, 
even when there is apparently no maternal problem.

1 Momotani N, Ito K, Hamada N, et al. Maternal hyperthyroidism and congenital malformation in the offspring. Clin Endocrinol (Oxf) 1984;20:695-700.

2 Momotani N, Noh J, Oyanagi H, et al. Antithyroid drug therapy for Graves' disease during pregnancy. Optimal regimen for fetal thyroid status. N Engl F Med 1986;315:24-8.

3 Mandel SJ, Brent GA, Lasson PR. Review of antithyroid drug use during pregnancy and report of a case of aplasia cutis. Thyroid 1994:4:129-33.

4 Wing DA, Millar LK, Koonings PP, et al. A comparison of propylthiouracil versus methinazole in the treatment of hyperthyroidism in pregnancy. Am $f$ Obstet Gynecol 1994;170:90-5.

5 Mitsuda N, Tamaki $\mathrm{H}$, Amino $\mathrm{N}$, et al. Risk factors for developmental disorders in infants born to women with Graves' disease. Obstet Gynecol 1992;80:359-64.

\section{A rare presentation of a common disease}

\section{Q1: What are the radiographic findings} (see p 662)?

The thoracic radiograph shows normal lung fields with subtle linear lucencies along the superior left cardiac border and in the paratracheal region consistent with a pneumomediastinum. The heart and trachea are outlined in the radiograph because air is radiolucent and it tracks along the fascial planes. There is also free intraperitoneal air most evident below the right hemidiaphragm. The abdominal radiograph shows similar radiolucent tracks outlining the psoas muscles. The abdominal computed tomogram confirms the presence of retroperitoneal air with extensive inflammation.

Q2: What is the most likely diagnosis?

Pneumomediastinum is usually caused by a perforation of the upper aerodigestive tract but it is rarely due to a leak from the infradiaphragmatic intestine. Perforation of any part of the aerodigestive tract may be traumatic, spontaneous, or iatrogenic in origin. The latter includes endoluminal or percutaneous procedures and positive pressure ventilation of the respiratory tract.

The presence of pneumomediastinum, pneumoperitoneum, and retroperitoneal inflammation suggests a perforation of the lower gastrointestinal tract. Without a history of recent trauma or invasive procedure, a spontaneous perforation of a colonic lesion into and posterior to the peritoneum is the most likely diagnosis. Diverticular disease is the most common cause of this unusual combination, although carcinoma and ulcerative colitis may rarely present this way.

\section{Q3: What are the treatment options?}

The decision between performing a primary anastomosis or bowel exteriorisation after resecting the affected colon depends on the extent of faecal contamination. The risk of anastomotic failure is high in the presence of sepsis and malnutrition. An ileostomy with mucous fistula formation for right sided and Hartmann's procedure for left sided colonic perforations are preferable to primary anastomosis if there is extensive soiling.

\section{Learning points}

- Colonic perforation occurs in about $15 \%$ of patients with complicated diverticular disease and is usually intraperitoneal.

- Retroperitoneal diverticular perforation is rare and is suggested by the presence of pneumomediastinum, subcutaneous emphysema, and/or pneumothorax.

- Resection of the perforated bowel with exteriorisation is usually required. Occasionally non-operative management is possible if the sepsis is contained but surgery becomes mandatory with clinical deterioration.

Attention should be paid to eliminating abdominal and mediastinal sepsis postoperatively. This requires intraoperative abdominal lavage, postoperative respiratory physiotherapy and broad spectrum intravenous antibiotics until bacterial sensitivities are available. Nutritional support is also essential in the perioperative period to reverse the catabolic state.

Localised abscess formation without septicaemia after a sealed colonic perforation may be treated conservatively. This includes adequate percutaneous drainage of the abscess, antibiotic therapy, oral abstinence, and parenteral nutrition to reduce colonic loading. Failure to progress or clinical deterioration mandates early surgical intervention.

This patient underwent a laparotomy where an abscess cavity was found in the left paracolic gutter in association with diverticulitis of the descending colon. The abscess cavity extended along the left psoas muscle cranially towards the mediastinum and caudally into the thigh. Crepitus was felt along the left psoas muscle. A Hartmann's procedure was performed due to the extent of the sepsis. Histological examination of the resected specimen confirmed the presence of a perforated colonic diverticulum. The patient subsequently died from multiorgan failure.

\section{Discussion}

Colonic diverticular disease is common and its complications can cause significant morbidity and mortality. Up to a quarter of patients with acute diverticulitis will require surgery and about $15 \%$ of these are due to colonic perforation. ${ }^{1}$ Most diverticula perforate only into the peritoneal cavity resulting in a localised pericolic abscess. This may subsequently fistulate into the bladder, adjacent bowel, or cutaneously. However generalised peritonitis supersedes if the sepsis is not contained with a high risk of septic shock and multiorgan failure. The mortality following a Hartmann's procedure for a left sided perforation is $12 \%$ compared with $28 \%$ for peritoneal drainage alone, with or without oversewing the mucosal defect. ${ }^{2}$

In contrast retroperitoneal colonic perforation due to diverticular disease is rare. ${ }^{34}$ The descending rather than sigmoid colon is more likely to perforate this way because the former is fixed in the retroperitoneum. Furthermore since diverticula form where the blood vessels 
penetrate the bowel wall and because the sigmoid colon has a free mesentery, a diverticulum at this site will generally perforate into the peritoneal cavity.

Signs of peritonitis may be absent if the sepsis is confined to the retroperitoneum and this can lead to a diagnostic delay. Indeed the sepsis may track downwards and present as a chronic abscess involving a hip joint ${ }^{5}$ or as thigh pain. ${ }^{6}$ In addition the high intra-abdominal and colonic pressure can lead to interstitial tracking of air upward to areas of low pressure resulting in pneumomediastinum. ${ }^{7}$ Subsequent rupture into the pleural cavity and subcutaneous tissue results in a pneumothorax ${ }^{8}$ and surgical emphysema respectively. ${ }^{4}$

The possibility of a colonic perforation should be considered in patients who present with pneumomediastinum, pneumoperitoneum, and retroperitoneal inflammation. While an anteroposterior thoracic radiograph may miss half of the cases of pneumomediastinum, a lateral view usually demonstrates retrosternal air. Abdominal and thoracic computed tomograms may help to confirm the diagnosis.

\section{Final diagnosis}

Colonic diverticular perforation resulting in pneumomediastinum, retroperitoneal inflammation, and pneumoperitoneum.

1 Tucci G, Torquati A, Grande M, et al. Major acute inflammatory complications of diverticular disease of the colon: planning of surgical management. Hepatogastroenterology 1996;43:839-45.

2 Roberts P, Abel M, Rosen L, et al. Practice parameters for sigmoid diverticulitis. The Standards Task Force American Society of Colon and Rectal Surgeons. Dis Colon Rectum 1995;38:125-32.

3 Andresen R, Meyer DR, Banzer D. [Soft tissue emphysema as manifestation of perforating diverticulitis]. Aktuelle Radio $1997 ; 7: 202-4$

4 Hur T, Chen Y, Shu GH, et al. Spontaneous cervical subcutaneous and mediastinal emphysema secondary to occult sigmoid diverticulitis. Eur Respir f 1995;8:2188-90.

5 Messieh M, Turner R, Bunch F, et al. Hip sepsis from retroperitoneal rupture of diverticular disease. Orthop Rev 1993; 22:597-9.

6 Haiart DC, Stevenson P, Hartley RC. Leg pain: an uncommon presentation of perforated diverticular disease. $\mathcal{F} R \mathrm{Coll}$ Surg Edinb 1989;34:17-20.

7 Suros J, Lee RA. Pneumoretroperitoneum, pneumomedistinum, and subcutaneous emphysema. Complications of acute, perforated diverticulitis. Minn Med 1973;56:747-9.

8 Tam WC, Pollard I, Johnson RD. Case report: pneumomediastinum and pneumothorax complicating colonoscopy. $\mathcal{F}$ Gastroenterol Hepatol 1996;11:789-92.

\section{Adrenal mass in a diabetic with hypergastrinaemia}

Q1: What other conditions besides Zollinger-Ellison syndrome could cause a raised gastrin level?

Causes of hypergastrinaemia are given in box 1.

Q2: Why does hypercalcaemia sometime present with abdominal pain?

There are various clinical manifestations of hypercalcaemia (see box 2). Abdominal pain associated with hypercalcaemia could be due to the manifestations of renal calculi, constipation, peptic ulcer disease (also associated with multiple endocrine neoplasia type 1 (MEN1) in patients with Zollinger-Ellison syndrome), pancreatitis, and enlargement of spleen in haematological malignancies.

Q3: How would you proceed to investigate whether the adrenal mass is functioning or just an incidental finding?

Adrenal tumours are common in MEN1 syndrome and have been reported in up to $36 \%$ of these patients. ${ }^{1}$ They are usually benign non-functioning adenomas and occur in association with pancreatic tumours.

Adrenal incidental mass is defined as an adrenal mass discovered by non-invasive abdominal imaging technique (computed tomography, magnetic resonance imaging, or ultrasound) performed for reasons other than suspected adrenal disease. ${ }^{2}$ The majority of adrenal incidental masses are benign nonsecretory adenomas.

A cost effective approach should be followed to decide whether the adrenal mass is secretory, and whether it is benign or malignant. Hormonal screening should include urinary catecholamines or metanephrines, urinary free cortisol and, in hypertensive patients, plasma renin activity and aldosterone level. ${ }^{2}$

Primary adrenal carcinoma is rare, whereas metastasis from pancreatic tumours to the adrenal is common. The relative frequency of primary adrenal carcinoma in adrenal incidental masses varies from $4 \%$ to $25 \%$ according to two different series. Features that suggest malignancy include tumour diameter of more than $6 \mathrm{~cm}$, haemorrhage, calcification, and necrosis.

Adrenal metastases vary in size, and tend to be smaller than the lesion of primary adrenal carcinoma and larger than those of adenoma. They are often bilateral.

Imaging techniques, scintigraphy, and fine needle aspiration biopsy are useful diagnostic tools in the evaluation of adrenal masses. Regular clinical and radiological follow up is

Box 1: Causes of hypergastrinaemia Achlorohydria

- Atrophic gastritis.

- Pernicious anaemia.

- $\mathrm{H}_{2}$ receptor antagonist.

- Proton pump inhibitors.

- Vagotomy.

- Gastric surgery (antrum retained).

Zollinger-Ellison syndrome

Hypercalcaemia

Box 2: Clinical manifestations of hypercalcaemia

- Neurological-lethargy, confusion, irritability, stupor.

- Psychiatric - depression, hallucination.

- Gastrointestinal - anorexia, nausea, vomiting, pancreatitis.

- Cardiovascular system-short QT interval, increase myocardial activity.

- Renal-nephrolithiasis, nephrogenic diabetes insipidus. 


\section{Box 3: Causes of incidental adrenal} masses

- Adrenal cortex-adenoma, nodular hyperplasia, carcinoma.

- Adrenal medulla-phaeochromocytoma, ganglioneuroma, neuroblastoma.

- Adrenal metastasis.

- Other adrenal masses-lipoma, neurofibroma.

- Pseudoadrenal masses-from kidney, spleen, or liver.

needed to monitor the progress of adrenal masses not needing immediate action. Causes of incidental adrenal masses are given in box 3 .

\section{Q4: Why did this man develop diabetes mellitus?}

The likely cause of diabetes in this patient is pancreatic insufficiency after surgery, although the time lapse between his surgery and development of diabetes is rather long. However, he was noted to have had impaired glucose tolerance five years after surgery. Autoimmune diabetes should also be considered, and can be screened for by checking anti-islet autoantibodies including glutamic acid decarboxylase antibodies.

MEN1 syndrome is associated with multiple pancreatic tumours, some of which are associated with diabetes mellitus, like glucagonomas and somatostatinomas. Our patient screened negative for pancreatic endocrine tumours other than gastrinoma.

Somatostatin analogues (for example, octreotide) are used for symptomatic relief in patients with pancreatic endocrine tumours. Octreotide inhibits insulin release, and sometimes impairs postprandial glucose tolerance. In rare instances with chronic adminstration, a state of persistent hyperglycaemia may be induced. ${ }^{3}$

\section{Q5: Why is it important to screen his family?}

This patient has the features of MEN1 syndrome, which is an autosomal dominant condition. Attempts to screen for the development of MEN1 tumours in asymptomatic relatives depend largely on measuring serum calcium, prolactin, and gastrointestinal hormones. Recent cloning of the MEN1 gene will enable earlier diagnosis and will possibly be used as a screening process for family members. ${ }^{4}$ Annual screening has been suggested from the age of 5 until the eighth decade in first and second degree relatives. Screening history and physical examination should be directed towards eliciting the symptoms and signs of hypercalcaemia, renal calculi disease, peptic ulcer disease, neuroglycopenia, galactorrhoea, amenorrhoea, Cushing's syndrome, visual field loss, and the presence of subcutaneous lipomas.

\section{Q6: Why did he develop a distal oesophageal stricture?}

Gastrin causes contraction of the lower oesophogeal sphincter/cardia, which is why oesophagitis and stricture formation is uncommon in Zollinger-Ellison syndrome. The presence of the nasogastric tube, after his initial surgery, coupled with his recumbent posture during recovery probably allowed reflux.

\section{Final diagnosis}

Multiple endocrine neoplasia type 1 (MEN1) syndrome with primary adrenal carcinoma.

1 Burgess JR, Greenway TM, Shepherd JJ. Expression of MEN-1 gene in a large kindred with multiple endocrine neoplasia type 1. F Intern Med 1998;243:465-70.

2 Mantero F, Arnaldi G. Investigation protocol: adrenal enlargement. Clin Endocrinol (Oxf) 1999;50:141-6.

3 Compendium of data sheets and product characteristics 1999-2000:1021-2.

4 Chadrasekharappa SC, Guru SC, Manickam P, et al. Positional cloning of the gene for the multiple endocrine neoplasia type 1. Science 1997;276:404-7.

Adult intussusception-an elusive diagnosis

Q1: The images show a characteristic mass lesion anteriorly. What diagnosis is suggested by these findings?

The findings are suggestive of intussusception. The images show a characteristic inhomogeneous soft tissue mass with fat stripes. ${ }^{1}$ When seen in longitudinal section, the mass may appear "sausage shaped" as in fig 1 (see questions; $p$ 663). When seen in cross section, the concentric loops of intussuscepted bowel produce a "target" appearance, as in fig 2 . This computed tomogram was consistent with an ileocolic intussusception.

\section{Q2: How may this condition present in adults?}

Intussusception in adults is unusual. An acute presentation is rare, more commonly the symptoms are insidious in onset and become chronic. The symptoms are variable and often non-specific. Abdominal pain is the most common symptom. ${ }^{2}$ Bowel obstruction is widely reported. ${ }^{3}$ Biliary obstruction ${ }^{4}$ and generalised peritonitis ${ }^{5}$ are also described.

Q3: How should such cases be managed? The preferred management of adult intussusception is surgical. Many cases involve neoplastic lead points. Malignant disease is more common where the colon is involved. In this case, a $25 \mathrm{~cm}$ ileocolic intussusception was identified at laparotomy. The lead point was a caecal tubulovillous adenoma. It was treated with an extended right hemicolectomy. The patient made a rapid recovery thereafter and was discharged home 10 days after surgery.

\section{Final diagnosis}

Intussusception.

1 Gayer G, Apter S, Hofmann C, Nass S, et al. Intussusception in adults: CT diagnosis. Radiology 1998;53:3-7.

2 Eisen LK, Cunningham JD, Aufses AH. Intussusception in adults: institutional review. $\mathscr{F}$ Am Coll Surg 1999;188:390-5. 3 Azar T, Berger DL. Adult intussusception. Ann Surg 1997; 226:134-8

4 Schnedl WJ, Reisinger EC, Lipp RW, et al. Biliary obstruction due to duodeno-jejunal intussusception in PeutzJeghers syndrome. Clin Gastroenterol 1996;23:220-3.

5 Navarro O, Daneman A, Alton DJ, et al. Colo-colic intussusception associated with pneumatosis cystoides intestinalis. Pediatr Radiol 1998;28:515-7. 
Acute respiratory failure in a middle aged woman

Q1: What is the differential diagnosis for this clinical presentation?

The differential diagnosis would include myasthenia gravis, Guillain-Barré syndrome, myotonic dystrophy, and mitochondrial myopathy.

The combination of peripheral and ocular muscle weakness together with the patient's history of other autoimmune conditions would be suggestive of myasthenia gravis as the underlying pathology. Although the condition has a peak age of onset at approximately 30 years, it is also associated with a later peak in more elderly age groups, often related to an underlying thymoma.

Guillain-Barré syndrome must be included because of the generalised muscle weakness and areflexia. The absence of sensory signs is also not atypical, although most cases are associated with a preceding viral illness. The Miller-Fisher variant should be considered but ataxia typically occurs in association with the ocular palsy.

Ptosis, muscle weakness, and a positive family history would prompt one to consider myotonic dystrophy as a possible diagnosis. Although 61 years would be considered old for a first presentation of previously unrecognised adult onset myotonic dystrophy, a milder condition can present after the age of $50 .{ }^{1}$ Ocular palsies, while previously described, are uncommon. ${ }^{2}$

Finally, mitochondrial diseases can be included because of their association with myopathies and neuropathies in a patient with a positive family history. Importantly, however, they are of maternal, not paternal, inheritance, as would be suggested in this case.

Q2: What does the electrocardiogram (fig 1 in questions; see $p$ 664) show and what is the significance of this?

There is a prolonged P-R interval of 0.24 $\mathrm{mm} / \mathrm{s}$, first degree heart block. This is of significance because first degree heart block is a common cardiac conduction defect identified in patients with myotonic dystrophy, ${ }^{3}$ its prevalence in the condition increasing with advancing age of the patient. As in this case, conduction defects are usually asymptomatic in myotonic dystrophy and should be screened for with an electrocardiogram.

Cardiac abnormalities are also features of both Guillain-Barré syndrome and myasthenia gravis. Guillain-Barré syndrome is associated with minor $\mathrm{T}$ wave changes in around $10 \%$ of cases and in myasthenia gravis, a prolonged Q-T interval and sinus arrhythmias are more typical. Cardiac features of mitochondrial diseases include cardiomyopathy and congestive cardiac failure.

Q3: What other clinical findings would be useful to elicit?

Other clinical signs which would be useful in obtaining a diagnosis include a slowly relaxing grip when the patient shakes hands, the classical sign of myotonia. Percussion myotonia can be demonstrated by tapping over the patient's thenar eminence, which induces contraction and slow relaxation of the abductor pollicis brevis muscle. Tongue myotonia may be demonstrated by tapping a spatula placed on the patient's tongue. Both grip myotonia and percussion myotonia were displayed in this case.

In a suspected case of myotonic dystrophy the examination should also include eye examination for cataracts and subcapsular deposits. Atrophy and weakness of the neck flexors should be assessed. Urinalysis and blood sugar testing should be performed to identify coexistent diabetes mellitus and in a male patient gynaecomastia and testicular atrophy should be examined for.

Where myasthenia gravis is suspected the demonstration of fatiguability is important.

\section{Q4: What further investigations would} you like to perform?

Useful tests to help differentiate between the conditions previously discussed are listed in box 1 .

The administration of edrophonium, a tensilon test, would be useful in helping to confirm myasthenia gravis. A significant improvement in the function of at least two muscle groups, as assessed by an independent observer would suggest the diagnosis.

Repetitive stimulation electromyography would be expected to show myotonic discharges and a reduction in the number of active motor units in a case of myotonic dystrophy. An electromyogram in this case was not useful in assisting with the diagnosis. Nerve conduction studies were within normal limits, suggesting that Guillain-Barré syndrome was unlikely in this case.

An autoantibody screen, including antiacetylcholine receptor antibody and antistriated muscle antibodies would also be necessary. In this patient neither antibody was positive.

A muscle biopsy would be helpful in distinguishing between myotonic dystrophy and mitochondrial disease. In our patient the muscle biopsy showed marked variation in fibre shape and size with focal fibre atrophy and hypertrophic fibres. There was an increased number of fibres with internal nuclei, many showed whorling (fig 1). ATPase staining showed type 1 fibre deficiency (fig 2). The

Box 1: Investigations of use in making a final diagnosis

- Tensilon test.

- Repetitive stimulation electromyography.

- Nerve conduction studies.

- Autoantibody screen -including antiacetylcholine and antistriated muscle antibodies.

- Muscle biopsy.

- Genetic analysis. 


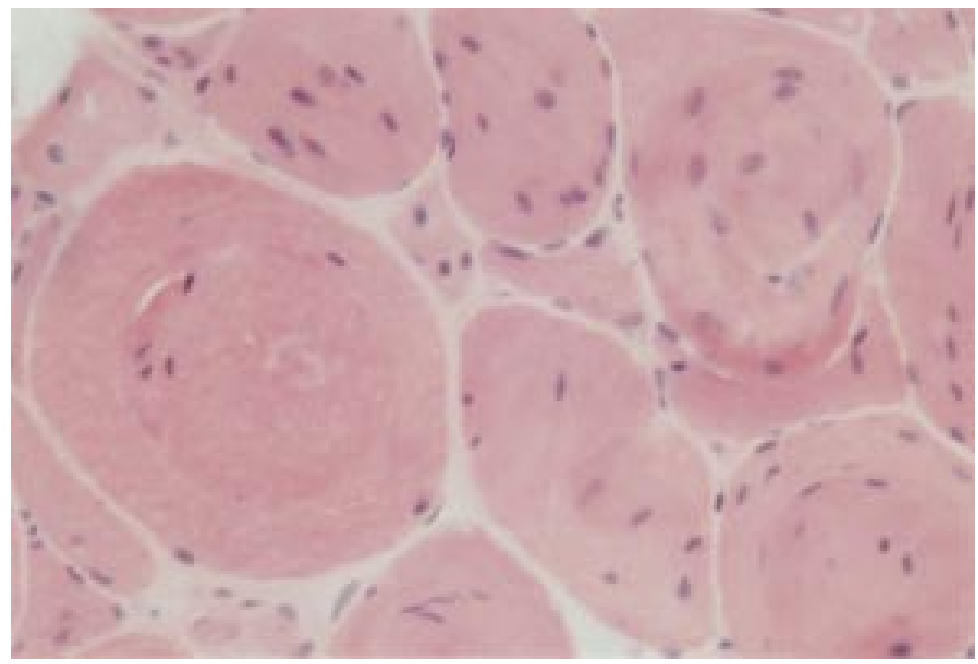

Figure 1 Haematoxylin and eosin staining of quadriceps muscle biopsy (magnification $\times 40$ ).

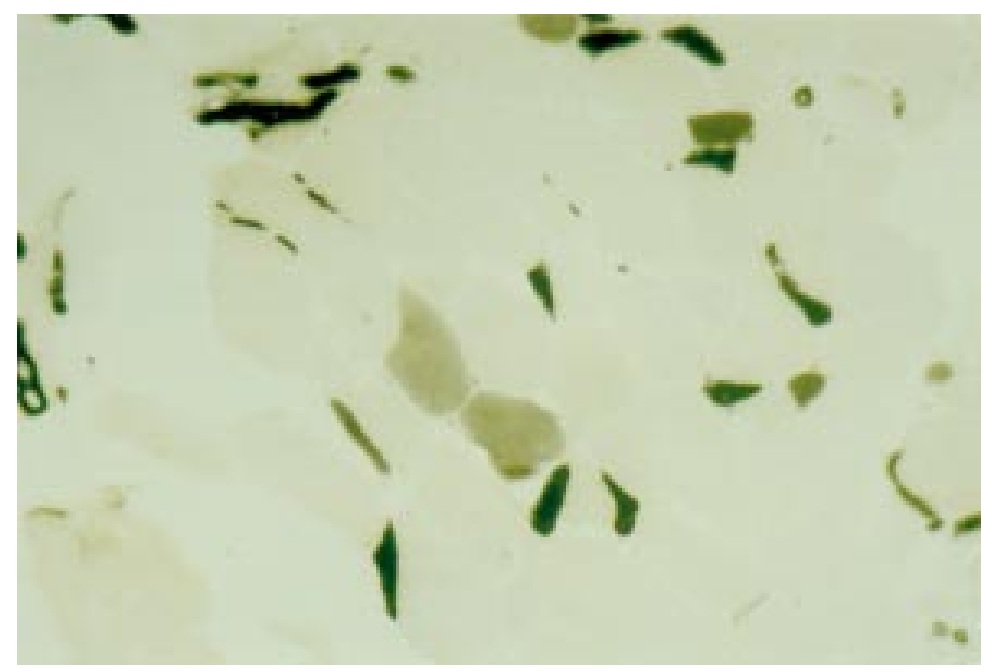

Figure 2 ATPase staining of muscle biopsy (magnification $\times 40$ ).

overall appearances were felt to be consistent with a diagnosis of myotonic dystrophy.

Once a histological diagnosis is made it should be confirmed with the help of genetic testing. The classical genetic defect responsible for myotonic dystrophy is an expanded cystosine-thymine-guanine (CTG) repeat in the 3' untranslated region of chromosome 19q13.3. ${ }^{4}$ In this patient genetic testing revealed the above abnormality confirming the final diagnosis to be myotonic dystrophy.

\section{Discussion}

This case illustrates an unusual cause of a common clinical presentation. Myotonic dystrophy is an autosomal dominant condition with multisystem involvement. It is the most common adult form of muscular dystrophy with an incidence of 13 per 100000 people. ${ }^{5}$ Many cases remain unrecognised so the true incidence is likely to be higher.

Myotonic dystrophy is caused by an expanded CTG trinucleotide repeat in the $3^{\prime}$ untranslated region of chromosome 19q13.3, the myotonic dystrophy protein kinase gene (DMPK). Normal individuals have between five and 37 repeats of the CTG sequence whereas individuals with myotonic dystrophy typically have greater than 100 such repeats. ${ }^{6}$ The length of the repeat correlates with both the age of onset and the severity of the condition. ${ }^{1}$ A proximal form of myotonic dystrophy has been described in which sufferers do not exhibit the CTG trinucleotide repeat in the DMPK gene but instead have an abnormality on chromosome $3 \mathrm{q}^{7}$

Adult myotonic dystrophy has a mean age of onset of 20-25 years of age. ${ }^{5}$ Clinically, it can present in a variety of ways to various specialties reflecting its multisystem involvement. Ultimately, as previously described, a combination of muscle weakness and myotonia allows the diagnosis to be made.

Muscle weakness can affect both striated and smooth muscles. Facial weakness and ptosis are often the earliest detectable features of the condition. Mild abnormalities may become apparent only by examining old photographs of the patient. Extraocular and jaw muscles can also become involved leading to the development of the classical myopathic facies. With advancing disease pharyngeal muscle involvement may lead to swallowing difficulties and an indistinct, nasal voice.

Muscle weakness in the limbs often begins distally, moving proximally with progression of the condition. Smooth muscle involvement produces many of the associated problems including dilatation and reduced motility in the oesophagus leading to aspiration, slowing of colonic movement resulting in constipation and megacolon and delayed emptying of the gallbladder predisposing to gallstone formation. $^{5}$

Selective degeneration of muscle fibres within the cardiac conducting system appear to be responsible for the arrhythmias and conduction defects which occur in myotonic dystrophy. Another documented feature of the condition is the development of premature senile cataracts. Myotonia should be sought in any young patient presenting to an ophthalmology clinic with cataracts in whom no obvious cause can be identified.

Myotonic dystrophy can generally be diagnosed on the basis of a clear history and thorough examination. In difficult cases such as the one discussed, a muscle biopsy will frequently be diagnostic. Histologically muscle fibres will classically show marked variation in size and shape. Type 2 fibres become hypertrophic and develop a "moth eaten" appearance while type 1 fibres become atrophic. An early change is the identification of an increased number of centrally placed nuclei in muscle fibres. Other typical changes include ringed fibres and homogenous areas of sacroplasm adjacent to them. ${ }^{5}$ Many of these changes are evident in the muscle biopsy of this patient (figs 1 and 2).

Prognostically, patients with adult onset myotonic dystrophy have a life expectancy of approximately 54 years. ${ }^{1}$ Myotonic dystrophy follows a progressive course with up to $14 \%$ of sufferers requiring a wheelchair due to motor disability before death. ${ }^{1}$ Pneumonia is the most frequent complication resulting in death. This is due to a combination of factors including 


\section{Box 2: Learning points}

- Myotonic dystrophy is the commonest muscle disease in adults.

- Inheritance is autosomal dominant and the genetic defect is a trinucleotide repeat expansion on chromosome 19q13.

- The size of the repeat expansion correlates with the age of onset an severity of the clinical course.

- Ocular palsies, although uncommon, are described.

- Grip and percussion myotonia are clinical hallmarks.

- Electromyography and muscle biopsy demonstrate characteristic changes and are useful tools in diagnosis.

- Cardiac conduction defects are common and cardiopulmonary complications are the commonest mode of death

- The availability of genetic screening and counselling for relatives is essential.

poor pharyngeal muscle function, delayed gastric and oesophageal emptying, weakness of respiratory and diaphragmatic musculature, and eventually decreased central respiratory drive. Treatment is directed at prevention of aspiration, aggressive antibiotic regimens, and ventilatory support if necessary.

Cardiac arrhythmias also account for a large proportion of deaths. Sudden death from cardiac problems in myotonic dystrophy appear to be independent from the severity of the condition. Frequent electrocardiograms are advised to detect conduction problems early. Elective cardiac pacing is of some benefit but sudden death can still occur in patients with permanent cardiac pacemakers in situ. ${ }^{1}$ Other important causes of death include fractures resulting from falls and postoperative complications.

At present no proven effective treatment is available for the underlying muscular problems in myotonic dystrophy, although drugs such as phenytoin and mexiletine have been used with limited benefit to improve myotonia. A recent pilot study on the use of intravenous dehydroepiandrosterone sulfate reported a benefit in improving muscle strength, decreasing myotonia, and improving cardiac conduction. Further controlled trials would be necessary before this becomes accepted treatment. ${ }^{8}$
Medical management is aimed at the detection and treatment of serious complications. Myotonic dystrophy remains an under diagnosed condition. We postulate that in this case the patient's father had myotonic dystrophy and died from a respiratory complication. Making the diagnosis early allows other family members to be offered the chance of genetic screening. Underlying neuromuscular disease in general and myotonic dystrophy in particular should always be considered in patients presenting with acute respiratory failure.

\section{Final diagnosis}

Myotonic dystrophy.

1 De Die-Smulders CEM, Höweler CJ, Thijs C, et al. Age and causes of death in adult onset myotonic dystrophy. Brain 1998;121:1557-63.

2 Anastasopoulos D, Kimmig H, Mergner T, et al. Abnormalities of ocular motility in myotonic dystrophy. Brain 1996;119:1923-32.

3 Hayashi $\mathrm{H}$, Ikeda U, Kojo T, et al. Cardiac conduction abnormalities and cytosine-thymine-guanine trinucleotide
repeats in myotonic dystrophy. Am Heart f 1997;134:292-7.

4 Harley HG, Rundle SA, Reardon W, et al. Unstable DNA

4 Harley HG, Rundle SA, Reardon W, et al. Unstable DNA
sequence in myotonic dystrophy. Lancet 1992;339:1125-8. sequence in myotonic dystrophy. Lancet 1992;339:1125-8.
Harper PS. Myotonic dystrophy. 2nd Ed. London: WB SaunHarper PS.
ders, 1989.

6 Simmon S, Thornton CA, Seltzer WK, et al. Relative stability of a minimal CTG repeat expansion in a large kindred with myotonic dystrophy. Neurology 1998;50:1501-4.

7 Ricker K, Grimm T, Koch MC, et al. Linkage of proximal myotonic myopathy to chromosome 3q. Neurology 1999;52: $170-1$.

8 Sugino $\mathrm{M}$, Ohsawa N, Ito T, et al. A pilot study of dihydroepiandrosterone sulfate in myotonic dystrophy. Neurology 1998;51:586-9.

\section{Cor pulmonale: a variation on a theme}

Q1: What investigations would help to identify the underlying cause of cardiac failure in this patient?

All patients with heart failure require identification of the underlying cause, so that treatment can be focused appropriately. In the present instance, electrocardiographic stigmata such as right bundle branch block, and a change in mean axis from its previous value of -15 degrees to its present value of +121 degrees (fig 1), suggested a diagnosis of pulmonary embolism, possibly recurrent, with consequent cor pulmonale. The coexistence of type 1 respiratory failure, with arterial oxygen and carbon dioxide partial pressures of $7.3 \mathrm{kPa}$ and $3.0 \mathrm{kPa}$, respectively, appeared to support this provisional diagnosis.
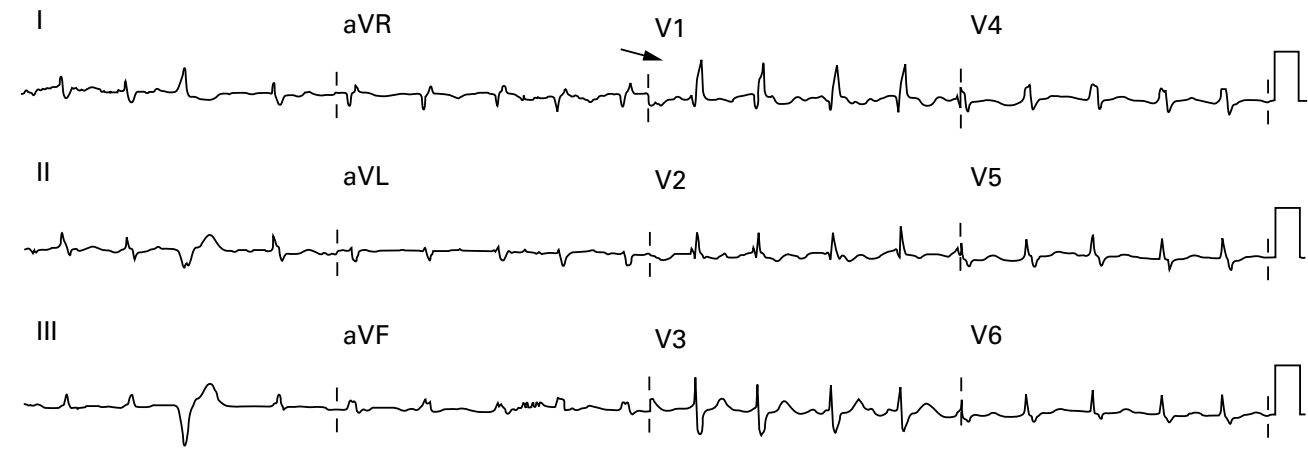

Figure 1 Electrocardiogram showing atrial fibrillation and right bundle branch block. 


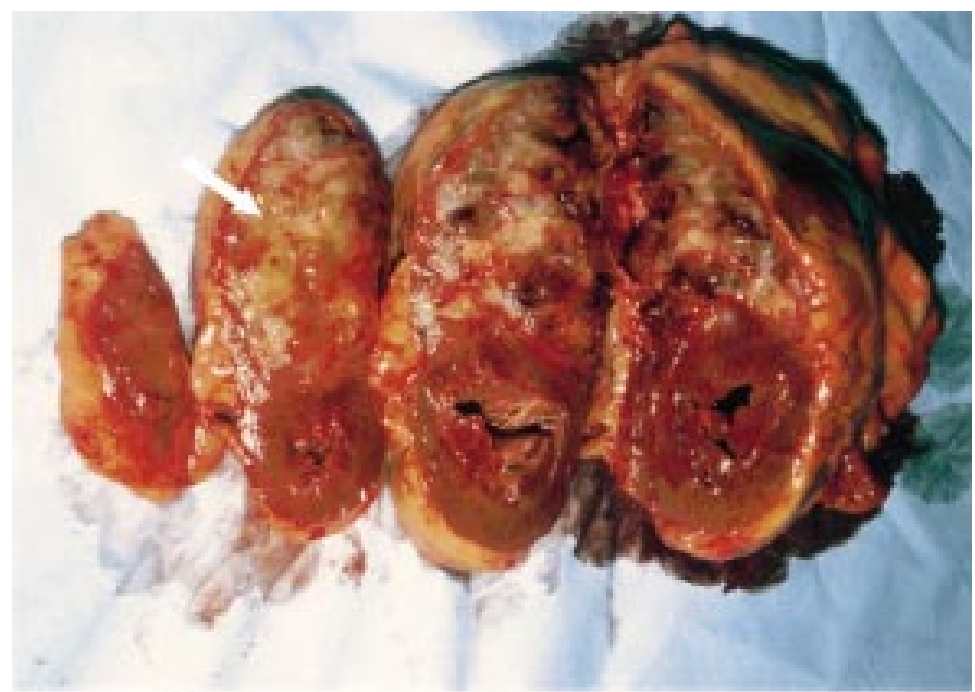

Figure 2 Necropsy specimen showing myxomatous tumour in the right ventricular cavity (arrowed).

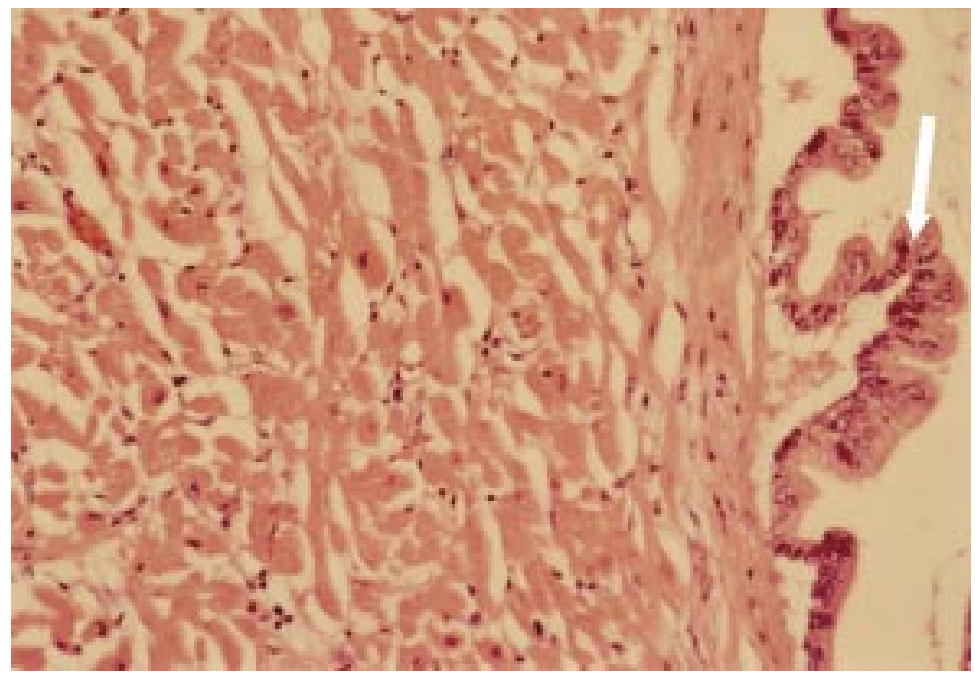

Figure 3 Histology of the right ventricular tumour showing papillary glands (arrowed) on the endocardial surface (haematoxylin and eosin; magnification $\times 200$ ).

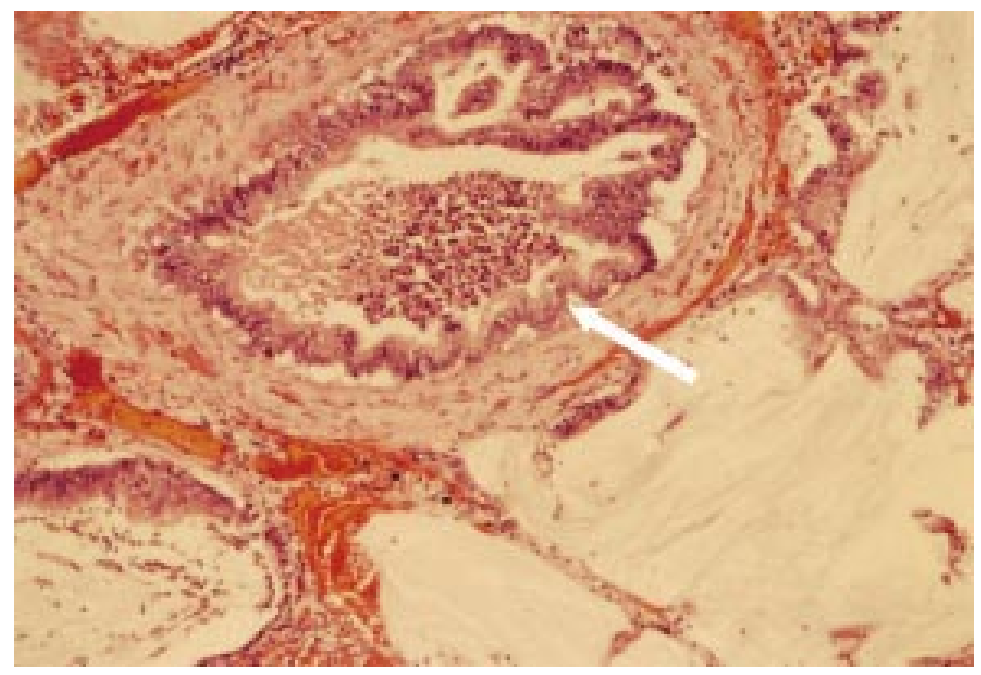

Figure 4 Histology of the metastatic tumour (arrowed) in the lumen of a pulmonary blood vessel (haematoxylin and eosin; magnification $\times 100$ ).
Q2: Could there be a link between the atrial fibrillation and evolution of the changes in the QRS axis?

When cor pulmonale complicates pulmonary thromboembolism it is typically characterised by type 1 respiratory failure, as in the present case. ${ }^{1}$ Atrial fibrillation, though more commonly complicated by systemic embolism (as a result of thrombus formation in the left atrial appendage), can occasionally be complicated by thrombosis within the right appendage, as in six of 90 patients with atrial fibrillation who had evaluable echocardiograms in the study reported by de Divitiis et al, ${ }^{2}$ pulmonary embolism being a potential complication. In the present instance, this was one of the potential aetiopathogenetic pathways for cor pulmonale.

\section{Treatment and progress}

She was anticoagulated and also treated with diuretics and continuous administration of oxygen. However, despite a good diuretic response, characterised by a $8 \mathrm{~kg}$ fall in body weight, she deteriorated, and died on day 54 .

\section{Postmortem findings}

Severe coronary heart disease was documented, and the right ventricle was shown to contain a myxoid tumour measuring $11 \times 10 \times$ $7 \mathrm{~cm}$ (see fig 2). The lungs were infiltrated by multiple nodules, up to $1.5 \mathrm{~cm}$ in diameter, composed of similar material. Histologically, the right ventricular tumour was composed of malignant glandular epithelium (see fig 3) with large, pleomorphic, and vesicular nuclei characterised by moderate mitotic activity. There had obviously been secretion of vast amounts of mucinous material. An acute inflammatory infiltrate also extended throughout the tumour. Tumour emboli were identified within the lumen of pulmonary blood vessels (see fig 4). Extrathoracic primary or secondary tumour could not be identified in the gastrointestinal tract, pancreas, ovary, uterus, thyroid, or elsewhere.

\section{Discussion}

On the basis of antemortem chest radiography, and antemortem echocardiography, this patient already had a provisional diagnosis of cor pulmonale attributable to pulmonary metastatic disease, ${ }^{3}$ with associated intracardiac mass of unknown origin. At necropsy, the crucial distinction was between primary and secondary cardiac neoplastic disease, the latter being by far the more common entity. ${ }^{4}$ Within the heart itself, malignant infiltration of the endocardium is much less likely than involvement of either the myocardium or the pericardium. ${ }^{5}$ The right ventricular endocardium is the logical site for metastases originating from the systemic circulation, as in colonic carcinoma, ${ }^{6}$ while blood borne metastases originating from the pulmonary circulation would first have to be seeded to a systemic location (for example, the adrenal gland), before metastasising to the right side of the heart. ${ }^{7}$ Exceptionally, as in the case reported by Ragle, a primary tumour in the right lung can spread to the right atrium, the carcinoma cells having been 
"carried by the systemic circulation, and not by the pulmonary circulation". ${ }^{8}$ Assuming that our patient, also, had a primary pulmonary tumour, a similar mode of spread could have accounted for secondary deposits in the right ventricle, with subsequent tumour embolisation to the rest of the lung. Although primary myxoma of the right ventricle ${ }^{9}$ seemed a plausible alternative diagnosis, with tumour embolism as a complication, ${ }^{10}$ the histological appearances were not typical, and the presence of mitotic activity also appeared to mitigate against this diagnosis. ${ }^{9}$

\section{Final diagnosis}

Cor pulmonale resulting from pulmonary tumour metabolism.

1 Fishman P. Cor pulmonale. In: Thorn GW, Adams RD, Barunwald E, et al, eds. Harrison's principles of internal Barunwald E, et al, eds. Harrison's principles

2 De Divitiis M, Omran H, Rabaieh R, et al. Right atrial De Divitiis $M$, Omran $\mathrm{H}$, Rabaieh $\mathrm{R}$, et al. Right atrial
appendage thrombosis in atrial fibrillation: its frequency and clinical predictors. Am f Cardiol 1999;84:1023-8.

3 Schriner RW, Ryu JH, Edwards WD. Microscopic tumour embolism causing subacute cor pulmonale: a difficult antemortem diagnosis. Mayo Clin Proc 1991;66:143-8.

4 Lan KY, Dickens P, Chan ACL. Tumours of the heart. A 20 year experience with review of 12485 consecutive autopsies Arch Pathol Lab Med 1993;117:1027-31.

5 Klatt EC, Heitz DR. Cardiac metastases. Cancer 1990;65 1456-9.

6 Anonymous. Case records of the Massachusetts General Hospital. Case 45-1992. N Engl f Med 1992;327:1442-8.

7 Norberts WC. Primary and secondary neoplasms of the heart. Am f Cardiol 1997;80:671-82.

8 Ragle HE. Implantation of carcinoma cells on the endocarRagle HE. Implantation of carcinoma cells on the endocar-
dium of the right auricle. United States Naval Medical Bulletin $1930 ; 28: 613-15$.

9 Reynen K. Cardiac myxomas. N Engl f Med 1995;333. Reynen $1610-17$.

10 Miyauchi Y, Endo T, Kuroki S, et al. Right atrial myxoma presenting with recurrent episodes of pulmonary embolism. Cardiology 1992;81:178-81.

\section{Rectal bleeding in a patient with portal hypertension}

Q1: What was the most probable cause of rectal bleeding in this woman?

The cause of rectal bleeding was rupture of rectal varices.

\section{Q2: What is the prevalence of this condition in portal hypertension?}

The prevalence of rectal varices in the patients with portal hypertension is quite high and varies from $43 \%-78 \%$. However bleeding from rectal varices is rare: the incidence is between $1 \%$ and $8 \%$.

\section{Q3: What is the management?}

There is no definitive guideline for the management of rectal variceal bleed. Conservative management may be all that is needed in mild cases and the bleeding may be self limiting. On the other hand it may be life threatening. Injection sclerotherapy, ligation of varices, transjugular intrahepatic portosystemic shunts (TIPS), and surgical portosystemic shunts have been tried with variable success.

\section{Discussion}

Bleeding from gastro-oesophageal varices is a life threatening complication of portal hypertension. Varices may also develop in other parts of the gastrointestinal tract. Large bowel is the second most common site of ectopic varices. The prevalence of this condition ranges between $43 \%$ and $78 \% .{ }^{1-3}$ The development of colorectal varices is dependent upon the degree of portal hypertension but does not depend upon its cause. ${ }^{4}$ Theoretically, obliteration of oesophageal varices either by banding or sclerotherapy may facilitate development of colorectal varices by increase in the blood flow through other portosystemic anastomoses. There is, however, no evidence to suggest that these treatment increase the prevalence of rectal varices.

Bleeding from rectal varices is rare. The incidence varies between $1 \%$ and $8 \% .{ }^{145}$ The severity may vary between mild to life threatening. Flexible sigmoidoscopy and colonoscopy can usually detect the varices but endoscopic ultrasound is more sensitive. Inferior mesenteric arteriography with special attention to the venous phase is very sensitive and is the diagnostic test of choice.

Conservative treatment with fluid replacement, blood transfusion, and correction of coagulopathy may be all that is needed in mild cases. Octreotide has been used to control severe variceal bleeding.

There is no consensus about the interventional management of rectal varices. Endoscopic ligation of the varices and injection sclerotherapy has been tried with variable success. ${ }^{6}$ Other methods that have been attempted are TIPS and surgical portosystemic shunt. The former can be complicated by stent occlusion and the latter by the development of hepatic encephalopathy. In some cases colectomy is the only option though this itself can be a major undertaking in advanced cirrhotic patients.

\section{Final diagnosis}

Bleeding from rectal varices.

1 Hosking SW, Smart HL, Johnson AG, et al. Anorectal varices, haemorrhoids, and portal hypertension. Lancet 1989;i:349-52.

2 Chawla Y, Dilawari JB. Anorectal varices-their frequency in cirrhotic and non-cirrhotic portal hypertension. Gut 1991;32:309-11

3 Wang TF, Lee FY, Tsai YT, et al. Relationship of portal pressure, anorectal varices and haemorrhoids in cirrhotic patients. F Hepatol 1992;15:170-3.

4 Goenka MK, Kochhar R, Nagi B, et al. Rectosigmoid varices and other mucosal changes in patients with portal hypertension. Am f Gasteroenterol 1984;79:756-60.

5 Ganguly S, Sarin SK, Bhatia V, et al. The prevalence and spectrum of colonic lesions in patients with cirrhosis and non-cirrhotic portal hypertension. Hepatology 1995;21: $1226-31$.

6 Levine J, Tahiri A, Banerjee B. Endoscopic ligation of bleeding rectal varices. Gastrointest Endosc 1993;39:188-91.

\section{A state of confusion}

Q1: What is illustrated in fig 1 (see p 666)? Scleromalacia-this condition results from severe scleritis and can lead to permanent localised thinning of the sclera, which develops a blue tinge. The sclera may go on to perforate-scleromalacia perforans; this complication results in blindness. 
Table 1 Range of organ involvement in rheumatoid vasculitis

\begin{tabular}{llll}
\hline \multirow{4}{*}{ Vessel type } & & \\
\cline { 2 - 4 } & Small vessels & Medium vessels & Large vessels \\
\hline Organ & Mononeuritis & Renal & Cardiac \\
& multiplex & Cutaneous & $\begin{array}{l}\text { Cerebral } \\
\text { Bowel }\end{array}$ \\
& Cutaneous & & \\
\hline
\end{tabular}

Q2: What is the skin lesion illustrated in fig 2 (see p 666)?

This is a vasculitic ulcer of the lower leg. It is most likely to be caused by rheumatoid arthritis in this patient, and is relatively uncommon in this condition.

\section{Q3: What is the unifying diagnosis?}

This patient had rheumatoid vasculitis, manifesting with mononeuritis multiplex of the left ulnar nerve, larger vessel involvement in the form of leg ulceration, and multiple areas of cerebral ischaemic change that resulted in the clinical presentation of a stroke. The scleromalacia affecting his eye was also another extra-articular manifestation of rheumatoid arthritis. Involvement of the larger arteries, in this case, the brain, is seen rarely. In this patient, a lumbar puncture revealed raised cerebrospinal fluid protein. His erythrocyte sedimentation rate and $\mathrm{C}$ reactive protein were also high, all indicators of active rheumatoid disease.

\section{Q4: What treatment would you now offer the patient?}

Aggressive treatment with cyclophosphamide is often required for the treatment of rheumatoid vasculitis involving medium to large sized vessels. Cyclophosphamide may be administered as a daily oral dose or an intermittent intravenous bolus infusion. Concomitant treatment with moderate doses of prednisolone are also sometimes required. The length of treatment is usually approximately six months, although some patients require maintenance therapy for years. Antibiotic treatment should be given for cutaneous infections. Foot and wrist drop are treated with splints.

\section{Q5: What is his prognosis?}

Prognosis depends on the organ system involved (see table 1). Patients with only limited cutaneous fingertip lesions generally do well. Involvement of major nerves or arteries in organs such as the heart are associated with a poorer prognosis.

\section{Discussion}

Fourteen previous cases have been reported in the literature of cerebral infarction complicating rheumatoid vasculitis. These cases have mostly been described as showing multiple large vessel infarctions in the cerebral hemisphere. Other large vessel involvement in rheumatoid vasculitis can result in infarction of the myocardium, bowel, lungs and very rarely, the kidneys. ${ }^{1}$ A diagnosis of rheumatoid vasculitis should be considered even if the patient does not have severe seropositive rheumatoid arthritis. The diagnosis of rheumatoid arthritis is often longstanding. Rheumatoid vasculitis is commoner in smokers. In cases of specific organ or tissue involvement such as a mononeuritis syndrome biopsy of the affected tissue (in this case the nerve) can be very useful in establishing the diagnosis. ${ }^{2}$ The aetiology of this condition lies in immune complexes, which probably contain rheumatoid factor. Immune complexes and are deposited in the walls of blood vessels. All patients with rheumatoid vasculitis have strongly positive titres for IgM rheumatoid factor. Most of the rheumatoid factor containing complexes can fix complement, which contributes to tissue damage.

\section{Final diagnosis}

Rheumatoid vasculitis.

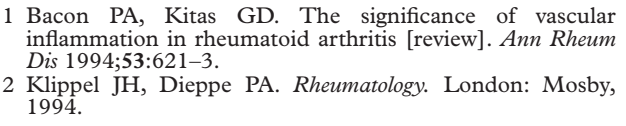

Dysphagia in a patient with palmoplantar keratoderma

Q1: What is the diagnosis?

Tylosis (palmoplantar keratoderma).

\section{Q2: How is it inherited?}

Autosomal dominant.

\section{Q3: What is the major complication?}

Oesophageal carcinoma.

\section{Discussion}

Tylosis is a rare hyperkeratotic condition characterised by thickening of the $\operatorname{skin}^{1}$ in those areas exposed to pressure and or friction as seen on the skin of the palms and soles (fig 1; see $\mathrm{p}$ 667). The condition is associated with itching and painful deep fissures. Family pedigrees indicate an autosomal dominant transmission pattern with a high degree of penetrance. There seems to be two forms of inherited tylosis: type $\mathrm{A}$ has a variable age of onset from 5 to 15 years; type B is manifested in the first year of life. ${ }^{2}$ The types run true in families and are not sex linked. Members of those families affected with tylosis have at least a $60 \%$ risk of oesophageal carcinoma by age 60 years. ${ }^{3}$ The paired conditions tylosis and oesophageal carcinoma are probably controlled at a single genetic locus.

\section{Pathogenesis of tylosis}

The actual pathological state maybe mediated through an increase in epidermal growth factor receptors (EGFRs) in the abnormal tissues. ${ }^{4}$ EGFRs have been found at increased levels in many malignant tumours but especially squamous cell carcinomas of the lung and oesophagus. $^{5}$

Because of the high risk of oesophageal carcinoma patients with tylosis require counselling and support. Obtaining a family history and pedigree is important in order to trace family members who may be unknown to the medical profession. Patients with this condition 
should be referred for specialist investigation for genetic evaluation.

When and how frequently these patients should undergo gastroscopy is presently unknown. In addition it is also unknown if modifying habits such as cessation of smoking and alcohol intake, prophylaxis with vitamin $A$, and treatment with proton pump inhibitors decrease the incidence of oesophageal carcinoma. It would seem logical for patients with tylosis to avoid other predisposing forces to oesophageal carcinoma-for example, mutagens (nitroso compounds, tannin from cigarettes) and promoters (phorbol esters present in some alcoholic beverages). If oesophagitis is one of the sequential steps in development of oesophageal carcinoma in patients with tylosis, then lifelong treatment with a proton pump inhibitor might well have a significant impact on oesophageal carcinoma developing.

\section{Final diagnosis}

Tylosis (palmoplantar keratoderma).

1 Cockayne EA. Inherited abnormalities of the skin. 1st Ed. London: Oxford University Press, 1933:188-95.

2 Harper PS, Harper RMJ, Howel-Evans AW. Carcinoma of the oesophagus with tylosis. $Q \mathcal{F}$ Med 1970;39:317-33.

3 Ellis A, Field JK, Field EA, et al. Tylosis associated with carEllis A, Field JK, Field EA, et al. Tylosis associated with car-
cinoma of the oesophagus and oral leukoplakia in a large cinoma of the oesophagus and oral leukoplakia in a large 7 Cancer 1994;30B:102-12.

4 Nanney LB, King LE, Dale BA. Epidermal growth factor receptors in genetically induced hyperproliferative skin disorders. Pediatr Dermatol 1990;7:256-65.

5 Nazmi MN, Dykes PJ, Marks R. Epidermal growth factor receptors in human epidermal tumours. Br $\mathcal{f}$ Dermatol 1990;123:153-61.

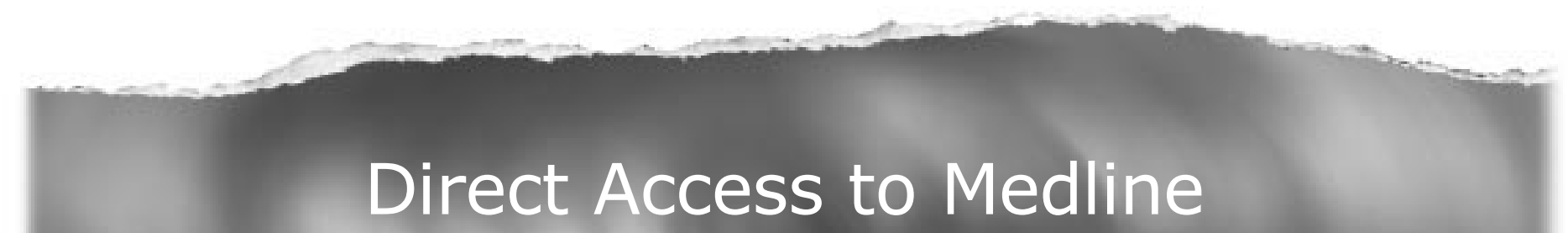

Medline

Link to Medline from the homepage and get straight into the National Library of Medicine's premier bibliographic database. Medline allows you to search across 9 million records of bibliographic citations and author abstracts from approximately 3,900 current biomedical journals.

\section{www.postgradmedj.com}

